\title{
Vergleichende Untersuchungen über die Strahlenempfindlidkkeit von Bacterium coli und von Hefe insbesondere bei Anwendung von energiereichen Strahlen
}

\author{
Von K. Sommermeyer und H. Magnus \\ Aus dem Radiologischen Institut der Universität Freiburg i. Br. \\ und dem Röntgeninstitut der Städt. Krankenanstalten Karlsruhe \\ (Z. Naturforschg. 15 b, 770-778 [1960] ; eingegangen am 23. Juni 1960)
}

Zur Prüfung, der insbesondere von Dobson (Deuteronenversuche mit B. coli) und von Houtermans (Temperaturversuche mit B. coli) und von ALPER (Betrachtungen über den RBW-Faktor bei Hefe) herrührenden Hypothesen, daß B. coli bei Anwendung von energiereichen Strahlen zum großen Teil durch strahleninduzierte inaktivierende Substanzen (IS) mit großen Diffusionslängen getötet wird und es sich bei der Abtötung von Hefezellen im wesentlichen um direkte Treffer in den lebenswichtigen Strukturen handelt, wurde eine vergleichende Untersuchung der Strahlenempfindlichkeiten von B. coli und haploiden Hefezellen vorgenommen. Dabei konnten die Auffassungen von Dobson, Houtermans und Alper bestätigt werden.

Bei Unterschreitung des Gefrierpunktes und Anwendung von $a$-Strahlen zeigen B. coli in Ubereinstimmung mit dem Befund Houtermans einen sprungartigen Anstieg der $D_{1 / 2}$. Hefe zeigt unter denselben Bedingungen nur einen allmählichen Anstieg.

B. coli kann man durch diffusible IS, die durch UV bei sehr hohen Dosen im Agar gebildet werden, praktisch vollständig zur Abtötung bringen, während sie auf haploide Hefezellen mit nahezu der gleichen Strahlenempfindlichkeit keine Wirkung ausüben.

Nach Bestrahlung des Agars sowohl mit UV als auch mit energiereichen Strahlen lassen sich, wenn man hohe Dosisleistungen anwendet, diffusible IS mit kurzen Lebensdauern $\left(T_{1 / 2}\right.$ bis zu etwa $15 \mathrm{sec})$ nachweisen. Der Reaktionsmechanismus bei Entstehung dieser IS (Mehrtreffer-Reaktionen) und bei ihrer Vernichtung im Agar erweist sich als äußerst kompliziert. Die Diffusionslänge der IS im Agar wurde zu rund $80 \mu$ bestimmt und ihr Diffusions-Koeffizient zu etwa $0,6 \cdot 10^{-5} \mathrm{~cm}^{2} / \mathrm{sec}$; dem entspricht ein mittleres Mol.-Gew. von rund 200. Die Ionenausbeute bei der IS-Bildung ist bei Anwendung von Röntgenstrahlen stark vom $\mathrm{O}_{2}$-Gehalt abhängig und bei Anwendung von $\alpha$-Strahlen unabhängig davon. Befindet sich der Agar in freier Atmosphäre, so ist die Ionenausbeute bei Anwendung der a-Strahlen 10-mal größer als bei Anwendung der Röntgenstrahlen. Die Zahl der zur Colizelle diffundierenden IS-Moleküle, die zu einer Abtötung notwendig ist, dividiert durch die Ionenausbeute bei ihrer Entstehung ist für Röntgenstrahlen von der Größenordnung $10^{9}$.

Die Bemühungen um eine eingehendere Beschreibung der primären Vorgänge bei der biologischen Strahlenwirkung haben ihren Ausdruck in prägnanter Form insbesondere in der Treffer-Theorie gefunden. Dort sind die primären Vorgänge Gegenstand der Theorie der Treffwahrscheinlichkeit. Die gegenwärtige Lage in dem ganzen Gebiet stellt uns vor die Aufgabe, unter verschiedenen als möglich erscheinenden primären Vorgängen die wichtigsten herauszufinden.

Der gesamte Ablauf der Strahlenreaktionen beginnt damit, daß besonders lebenswichtige Strukturen in der Zelle (vor allem die Chromosomen-Oberfläche oder die Chromosomen selbst) durch einen Treffer geschädigt werden, wobei die Theorie der Treffwahrscheinlichkeit drei verschiedene Arten von Treffern, d. h. also drei verschiedene Arten von primären Vorgängen unterscheidet:

a) direkte Treffer (Korpuskeldurchgänge durch die lebenswichtigen Strukturen).

b) Schädigung der Strukturen durch Wasserradikale oder deren unmittelbaren Folgeprodukte, welche in der unmittelbaren Umgebung der lebenswichtigen Strukturen entstehen und deren Diffusionswege im Plasma sehr kurz (etwa $<100 \AA$ ) sind.

1 K. Sommermeyer, in: Schinz, Holthusen, Langendorff, RAJewski, Schubert, Strahlenbiologie, Strahlentherapie, Nuk- c) Schädigung der lebenswichtigen Strukturen durch aktive organische Moleküle mit beträchtlichen Diffusionslängen im Plasma, also durch Moleküle, die aus beträchtlichen Entfernungen zu den biologisch wichtigen Strukturen herandiffundieren können (z. B. organische Peroxyde oder Peroxydradikale).

Zur Erforschung dieser verschiedenen Möglichkeiten für das Zustandekommen eines Treffers wird schon seit langem die Inaktivierung (Abtötung) von Einzellern durch energiereiche Strahlung untersucht und hierbei war insbesondere das mächtigste Hilfsmittel die Variation der Strahlenarten, in dem von Korpuskeln (eingeschossenen oder durch Strahlung in der Zelle ausgelösten) mit geringer spezifischer Ionisation, z. B. von energiereichen Elektronen, auf Strahlen mit großer spezifischer Ionisation, im Grenzfall auf $\alpha$-Strahlen, übergegangen wurde. Die beiden bekanntesten Objekte, mit denen viele solche Untersuchungenen ausgeführt wurden, sind Bacterium coli und Hefe, wir wollen uns auch im folgenden auf diese beiden Objekte beschränken.

In einem zusammenfassenden Bericht ${ }^{1}$ über den heutigen Stand der Treffertheorie haben wir uns kürzlich,

learmedizin, Krebsforschung, Ergebnisse 1952-1958, Stuttgart 1959. 
was die Inaktivierung von Bacterium coli anbetrifft, der Auffassung von DoBson angeschlossen; hinsichtlich der Inaktivierung von Hefe sind wir der Auffassung von Alper gefolgt.

a) B. coli. Dobson ${ }^{2}$ findet beim Utbergang von Röntgenstrahlen zu 190-MeV-Deuteronen einen Anstieg der Inaktivierungsdosen, die Inaktivierungsdosen IAD bzw. Halbwertsdosen $D_{1 / 2}$ fallen jedoch auf etwa $75 \%$ wieder $\mathrm{ab}$, wenn man von den 190-MeV-Deuteronen zu Deuteronen von $26 \mathrm{MeV}$ übergeht, um bei Anwendung von a-Strahlen wiederum um etwa $70 \%$ anzusteigen. Der Anstieg der Inaktivierungsdosen beim Übergang von Röntgenstrahlen zu 190-MeV-Deuteronen wird von DoBson als „Sättigungseffekt" gedeutet und angenommen, daß es sich bei den Röntgenstrahlen um direkte Treffer oder um die Wirkung von Radikalen mit sehr kurzen Diffusionswegen handelt. Der Abfall der $D_{1 / 2}$ beim Utbergang von $190-\mathrm{MeV}$ - zu 26-MeV-Deuteronen wird durch die Annahme erklärt, daß mit steigender spezifischer Ionisation im steigenden Maß IS mit sehr großen Diffusionslängen gebildet werden. Deren Bildung und Wirkung soll bei den $\alpha$-Strahlen vollkommen überwiegen und im übrigen der Anstieg der $D_{1 / 2}$ bei den $a$-Strahlen wiederum auf „Sättigungseffekt" beruhen. In bester Übereinstimmung mit der Auffassung von Dobson stehen auch Reaktivierungs-Versuche von LANGENDORFF und SoMmermeyer an einem sehr strahlenempfindlichen Colistamm und vor allem Versuche von Houtermans ${ }^{3}$ über die Abhängigkeit der $D_{1 / 2}$ von der Temperatur. Nach Houtermans nimmt bei Anwendung von Röntgenstrahlen, wenn man bei Abkühlung den Gefrierpunkt unterschreitet, die $D_{1 / 2}$ nur allmählich mit abnehmender Temperatur zu, während bei den $\alpha$-Strahlen die $D_{1 / 2}$ beim Gefrierpunkt sprungartig auf den doppelten Wert ansteigt. Der allmähliche Anstieg der $D_{1 / 2}$ bei den Röntgenstrahlen mit abnehmender Temperatur ist mit einer allmählichen Entwässerung der lebenswichtigen biologischen Strukturen oder mit einem verzögerten Gefrieren des Wassers in der unmittelbaren Umgebung der biologischen Strukturen in Zusammenhang zu bringen, während der sprungartige Anstieg der $D_{1 / 2}$ bei den $a$-Strahlen am einfachsten damit erklärt wird, daß im Plasma selbst IS mit großen Diffusionswegen gebildet werden, deren Bildung in festem Zustand z. T. unterdrückt ist.

b) Hefe. Hefe zeigt eine Abnahme der $D_{1 / 2}$ mit steigender spezifischer Ionisation, diese beginnt bei einer linearen Energieübertragung von etwa $20 \mathrm{KeV} / \mu$. Da die Abhängigkeit der radiochemischen Reaktionen in

2 B. L. Dosson, UCRL 1951, 1140.

8 Tн. Houtermans, Z. Naturforschg. 9 b, 601 [1954].

4 T. Alper, Rad. Res. 5, 573 [1956].

* Wir gehen nicht ein auf die umfangreiche Literatur über die Wirkung von Schubtzsubstanzen und auch nicht auf die Reaktivierung und auch auf den Sauerstoffeffekt erst weiter unten (Literatur hierüber unter l. c. ${ }^{5}$ ), weil unserer Meinung nach wenigstens zur Zeit alle diese Befunde sowohl mit indirekter Strahlenwirkung und großen Dif.fusionswegen als auch mit direkter in Zusammenhang gebracht werden können. wäßrigen Lösungen von der linearen Energieübertragung schon etwa bei $5 \mathrm{KeV} / \mu$ einsetzt, kann die Erklärung des $D_{1 / 2}$-Anstieges nach Alper schwerlich in der Radiochemie des Plasmas selbst gesucht werden. Es ist vielmehr nach ALPER ${ }^{4}$ wahrscheinlich, daß im wesentlichen direkte Strahlenwirkung auf die Hefechromosomen vorliegt, und zwar ein klassischer Konzentrationseffekt (d.h. die Inaktivierung geht mit weit größerer Wahrscheinlichkeit vor sich, wenn gleichzeitig mehrere primäre Ionisationen im strahlenempfindlichen Volumen d. h. im Hefechromosom oder seiner nächsten Umgebung erfolgen *.

\section{Versuchsprogramm}

Bei B. coli soll also nach Dobson, Langendorff und Sommermeyer und Houtermans die Bildung von IS mit sehr großen Diffusionswegen eine beträchtliche Rolle spielen, während bei Hefe nur direkte Treffer oder Treffer in der nächsten Chromosomenumgebung zur Wirksamkeit kommen sollen. Diese gesamte Auffassung ist offenbar einer eingehenderen experimentellen Nachprüfung zugänglich, und zwar

1. durch einen weiteren Ausbau der Temperaturversuche. Es ist notwendig, Temperaturversuche derart, wie sie Houtermans mit $B$. coli vorgenommen hat, auch mit Hefezellen auszuführen ${ }^{* *}$. Bestehen die Interpretationen von Houtermans und AlPER zu Recht, so darf sich bei Hefe unterhalb des Gefrierpunktes ein Unterschied in der Abhängigkeit der Inaktivierungsdosis von der Temperatur einmal bei Röntgenstrahlen, zum anderen bei $\alpha$-Strahlen in der Art, wie er bei $B$. coli gefunden wurde, nicht ergeben.

2. Durch Versuche mit vorbestrahltem Nährboden. Diffusible IS (oder mutagene Substanzen) mit großen Diffusionslängen kann man durch Bestrahlung von Nährböden herstellen und es ist insbesondere schon lange bekannt, daß B. coli (ebenso wie andere Bakterien) inaktiviert wird, wenn man es auf Nährböden aufbringt, die mit UV vorbestrahlt wurden ${ }^{6}$. Wir interessieren uns jedoch weniger für die inaktivierende Wirkung von Nähr-

5 J. Gagger u. G. E. Stapleton, 2. Congresso internationale die Fotobiologia 1957, Turin 1957; M. R. ZeLLe, S. 155 ; W. Harm u. W. Stein, Z. Naturforschg. 8 b, 742 [1953]; R. LatarJet, Strahlentherapie 101, 580 [1956] ; 110, 443 [1959].

* Solche Versuche wurden bisher bei Hefe zwar mit Röntgenstrahlen (vgl. l. c. ${ }^{1}$ ), aber nicht mit $\alpha$-Strahlen aus geführt.

6 O. Wyss, J. B. Clark, F. HaAs u. W. Stone, J. Bacteriol. 56, 51 [1948] ; Amer. Naturalist 84, 261 [1950], dort Angaben über ältere Literatur; W. STEIN u. W. HARM, Naturwissenschaften 39, 383 [1952]. 
böden, die mit UV vorbestrahlt sind, als für die inaktivierende Wirkung einer Vorbestrahlung mit energiereichen Strahlen. Merkwürdigerweise findet man hierüber in der Literatur überhaupt keine quantitativen Untersuchungen. (Die einzige qualitative Untersuchung s. 1. c. ${ }^{7}$.)

Die Klärung folgender Fragen ist von Interesse:

a) B. coli. Bei direkter Bestrahlung soll nach Dobson die Wahrscheinlichkeit der Entstehung von IS mit großen Diffusionslängen bei gleicher Dosis („Ionenausbeute“ der Reaktion) merklich zunehmen, wenn man von Röntgenstrahlen zu $\alpha$-Strahlen übergeht. Außerdem ist bekannt, daß bei B. coli (wie ganz allgemein bei der biologischen Strahlenwirkung) wohl bei Anwendung von Röntgenstrahlen die Inaktivierungsdosis vom Sauerstoffgehalt der Zellen abhängt aber nicht (oder nur sehr wenig) bei Anwendung von $\alpha$-Strahlen *. Es sind also eingehender die Bedingungen zu untersuchen, unter denen ein vorbestrahlter Nährboden das auf ihn aufgebrachte B. coli inaktiviert. Wenn die in dem Agar entstehenden diffusiblen IS identisch oder wesensgleich sind mit den in der Colizelle bei $\alpha$-Durchgängen entstehenden Giften, so ist zu erwarten, daß die Ionenausbeute an IS im Agar bei Anwendung von $\alpha$-Strahlen wesentlich größer ist als bei Anwendung von Röntgenstrahlen und außerdem bei den $\alpha$-Strahlen unabhängig von dem Sauerstoffgehalt des Nährbodens.

b) Die Versuche sind schließlich mit Hefe zu wiederholen und auch die Inaktivierung von Hefe auf vorbestrahlten Nährböden zu untersuchen. Die Wahrscheinlichkeit der Inaktivierung durch die diffusiblen Substanzen ist dann beim B. coli und Hefe $\mathrm{zu}$ vergleichen. Bei Hefe sollte die InaktivierungsWahrscheinlichkeit bei einer vorgegebenen ISMenge zumindest viel kleiner sein als bei $B$. coli.

\section{Durchführung der Versuche}

Versuchsbedingungen. Benutzt wurde B. coli vom Stamm 026 und ein haploider Hefestamm **. Der Agar hatte eine Zusammensetzung von 2,5\% Pronagar *, $0,3 \% \mathrm{NaCl}, 1 \%$ Pepton und $1 \%$ Fleischextrakt, B. coli wurde in der üblichen Weise in einer feinen Verteilung aus einer Nährbouillon auf die Agarplatten aufgebracht und die Hefezellen aus einer wäßrigen Suspension.

\footnotetext{
* Vgl. hierzu Dosson, Abb. 6.

** Wurde uns freundlicherweise von Herrn Professor WeINfortner (Institut für Mikrobiologie T.H. München) zur Verfügung gestellt.
}

Nach mikroskopischen Beobachtungen beginnt bei B. coli die logarithmische Wachstumsphase etwa 9 Min. nach dem Aufbringen auf den Agar. Die gebildeten Kolonien wurden nach 2 Tagen gezählt (Bebrütungstemperatur $37^{\circ} \mathrm{C}$ ).

Angewandt wurde eine Röntgenstrahlung von $30 \mathrm{keV}$ max. und einer HWS im Agar von etwa $1,2 \mathrm{~mm}$. Als $\alpha$-Strahler dienten Polonium-Plattenpräparate von etwa $2 \mathrm{~cm}$ Durchmesser, die in 1-2 mm Entfernung von der Agaroberfläche gebracht wurden. Gelegentlich wurden auch Versuche mit UV ausgeführt. Als UV-Quellen dienten dabei eine Osramlampe HNS 12 bei der vorwiegend die Linie 2537 Å emittiert wird.

$$
\begin{gathered}
\text { 1. Die Abhängigkeit } \\
\text { der Strahlenempfindlichkeit }
\end{gathered}
$$
von der Temperatur bei B. coli und Hefe

Die Dosisleistung war bei diesen Versuchen etwa von der Größe $10000 \mathrm{rad} /$ Minute. Die Versuche wurden nach der gleichen Technik durchgeführt wie bei HouterMANS.

Die Agarscheibe befand sich auf einer Kupferunterlage, die wiederum auf einem Kupferstiel saß, welcher in flüssiger Luft, Kochsalz-Eis-Mischungen oder $\mathrm{CO}_{2}$ Schnee endigte. Das Ergebnis der Versuche zeigt Abb. 1. Man erkennt, daß bei B. coli genau das Ergebnis von Houtermans reproduziert wurde, nämlich: es ergab sich bei Röntgenstrahlen ein langsamer Anstieg der Inaktivierungsdosis mit abnehmender Temperatur und bei a-Strahlen ein unstetiger Sprung beim Gefrierpunkt. Ganz anders ist das Resultat bei der Hefe, und zwar wie erwartet. Hier erhält man sowohl bei Anwendung von Röntgenstrahlen als auch von $a$-Strahlen einen allmählichen Anstieg der Inaktivierungsdosis mit abnehmender Temperatur.

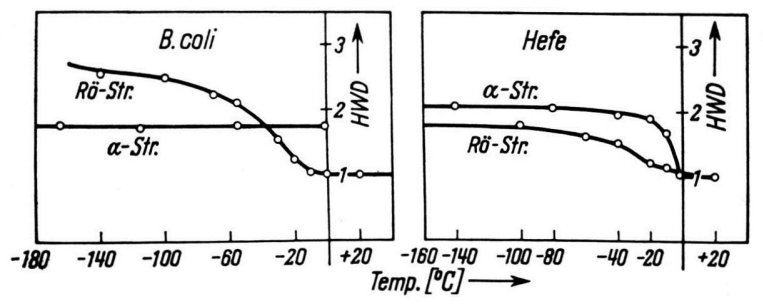

Abb. 1. Temperatur-Versuche.

2. Die Wirkung von vorbestrahltem Agar a uf $B$. coli

a) Die Entstehung der diffusiblen IS im Agar bei Bestrahlung in freier Atmosphäre mit Röntgenstrahlen, a-Strahlen und $U V$

Bei den Versuchen mit Röntgenstrahlen, Polonium- $a$ Strahlen und mit UV, wurden die Colibakterien zunächst unmittelbar (innerhalb einer Zeitdifferenz von

7 J. H. Blank u. H. Kersten, J. Bacteriol. 30, 21 [1935].

* Sämtlicher in der Arbeit benutzter Pronagar stammte aus der gleichen Lieferung. 
wenigen sec) nach der Bestrahlung aufgebracht und dann in üblicher Weise nach 2 Tagen die Kolonienzahl bestimmt. Bei den Versuchen wurde auch die Dosisleistung im Verhältnis $1 \mathrm{zu} 10$ variiert, und zwar betrug sie bei Röntgenstrahlen $12000 \mathrm{r} / \mathrm{min}$ bzw. $1200 \mathrm{r} / \mathrm{min}$, bei den $a$-Strahlen $7.000 \mathrm{rad} / \mathrm{min}$ und $700 \mathrm{rad} / \mathrm{min}$ und bei UV $1800 \mathrm{erg} / \mathrm{cm}^{2} \mathrm{~min}$ und $180 \mathrm{erg} / \mathrm{cm}^{2} \mathrm{~min}$.

Die Versuche hatten als hervorstechendstes Ergebnis (vgl. Abb. 2), daß die Zahl der Geschädigten zunächst angenähert linear mit der Dosis zunimmt, es gelingt aber keineswegs durch weitere Steigerung der Dosen alle Colibakterien abzutöten, vielmehr erreicht die Zahl der Geschädigten mit steigenden Dosen einen Grenzwert. So ergibt sich für Röntgenstrahlen bei einer Dosisleistung von $1200 \mathrm{r} / \mathrm{min}$ bei Steigerung der Dosis ein Grenzwert der Überlebensrate von $75 \%$, für $\alpha$-Strahlen bei einer Dosisleistung von $700 \mathrm{rad} / \mathrm{min}$ ein Grenzwert der Überlebensrate von $73 \%$ und bei UV bei einer Intensität von $180 \mathrm{erg} / \mathrm{cm}^{2}$ min ein Grenzwert der Úberlebensrate von 70 Prozent. Vermehrt man die Dosisleistung auf den 10-fachen Betrag, so liegt der Grenzwert der Überlebensrate bei einem nur wenig tieferen Wert, nämlich bei den Röntgenstrahlen bei etwa $52 \%$, bei den $\alpha$-Strahlen bei etwa $48 \%$ und bei den UV-Strahlen bei etwa 43 Prozent. Die Ergebnisse sind in Tab. 1 und Abb. 2 dargestellt.

Der Grund dafür, daß trotz weiterer Steigerung der Dosis ein so beträchtlicher Prozentsatz an Überlebenden übrig bleibt, kann nicht darin gesucht werden, daß die bestrahlten Colibakterien extreme Verteilung in der Variabilität ihrer Strahlenempfindlichkeit besitzen, sondern sie beruht darauf, daß die Menge der in Agar gebildeten IS mit steigender Dosis einem Gleichigewichtswert zustrebt, der dadurch gegeben ist, daß im Agar in der Zeiteinheit genausoviel IS verschwinden wie gebildet werden*. Der Beweis dafür, daß die IS im Agar nicht nur bei der Bestrahlung gebildet werden, sondern, daß mit der Erzeugung auch eine Vernichtung konkurriert, ergibt sich durch einen weiteren Versuch: Wenn man die Bakterien nicht unmittelbar nach der Bestrahlung auf den Agar bringt, sondern erst nach Beendigung der Agarbestrahlung eine bestimmte Zeit verstreichen läßt, so geht die Schädigung des aufgebrachten Coli bis auf einen gewissen Restbetrag ** rasch zurück, woraus hervorgeht, daß nach Aufhören der Bestrahlung die IS im Agar rasch verschwinden (vgl. hierzu Abb. 2, rechte Seite). In grober Näherung kann diese Abnahme mit der Zeit durch eine Exponentialfunktion dargestellt werden. Die Halbwertszeiten des Abklingens sind abhängig von der angewandten Dosisleistung, und zwar sind die Halbwertszeiten desto größer, je größer die Dosisleistung ist. Man erhält mit Rö.-Strahlen bei der Dosisleistung $1200 \mathrm{r} / \mathrm{min}$ und mit

* Speziell für Mutationen in Colisuspensionen nach vorheriger UV-Anwendung war dies bereits von Wyss und Mitarbb. gefunden worden.

** Es werden also in Agar auch langlebige IS-Moleküle gebildet. Der Prozentsatz der langlebigen IS-Moleküle liegt bei den Röntgenstrahlen, wie aus der Abb. 2 hervorgeht, zwischen 20 und 30 Prozent. Er ist bei den $\alpha$-Strah-

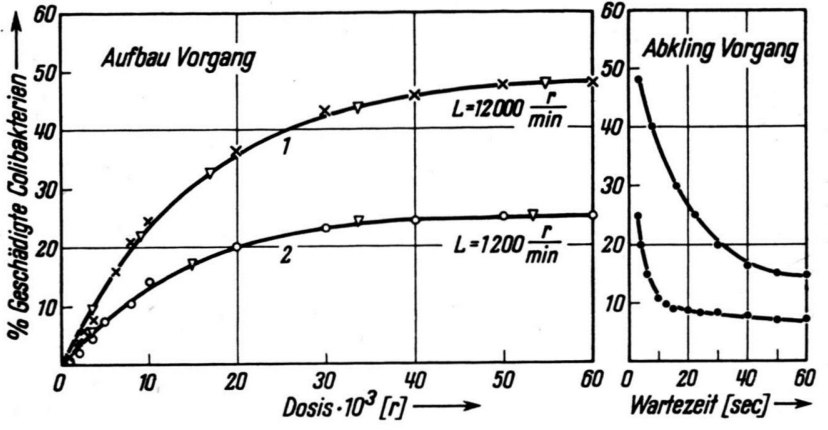

Abb. 2. Bildung des IS-Stoffes bei Anwendung von Rö-Strahlen.

$\begin{array}{rr}\text { Kurve 1. } \mathrm{B}=0,9 \cdot 10^{-4}(1 / r) & \text { Kurve 2. } \mathrm{B}=0,9 \cdot 10^{-4}(1 / r) \\ \mathrm{D}=7,2 \cdot 10^{-4}(1 / r) & \mathrm{D}=7,2 \cdot 10^{-4}(1 / r) \\ \mathrm{C}^{*}=7,6 \cdot 10^{-4}(1 / r) & \mathrm{C}^{*}=15,2 \cdot 10^{-4}(1 / r) \\ \text { theoret. Werte } \times & \text { theoret. Werte } \circ \\ \text { exp. Werte } \nabla & \text { exp. Werte } \nabla\end{array}$

a-Strahlen bei der Dosisleistung von $700 \mathrm{rad} / \mathrm{min}$ und bei UV bei einer Strahlenintensität von $180 \mathrm{erg} / \mathrm{cm}^{2} \mathrm{~min}$ eine Halbwertszeit von etwa 6 Sekunden. Wenn man die Dosisleistungen um den Faktor 10 vergrößert, verzögern sich die Halbwertszeiten für das Abklingen auf etwa 12 Sekunden.

Man könnte vielleicht zunächst vermuten, daß die ISMoleküle durch eine einfache Eintreffer-Reaktion nach $\mathrm{d} N / \mathrm{d} r=A$ gebildet werden und durch Reaktionen mit Bestandteilen des Agars wieder nach der Beziehung $N=N_{0} e^{-C t}$ verschwinden, wobei die Halbwertszeit des Vernichtungsprozesses $0,69 / C$ aus den vorher beschriebenen Versuchen über das Abklingen der IS nach Beendigung der Bestrahlung zu entnehmen sind. Damit würde sich für die Konzentration der IS mit steigender Dosis $N=A / C^{x} \quad\left(1-e^{-C^{\mathbf{x}} r}\right)$ mit $C^{x}=C / L$ ergeben, wobei $L$ die Dosisleistung ist, d. h. der Anstieg der IS mit der Bestrahlungszeit müßte die gleiche Halbwertszeit besitzen wie der Abfall der IS im Agar nach Beendigung der Strahlung. In Wirklichkeit ist dies aber keineswegs der Fall, sondern die Entstehung der IS, d. h. der zeitliche Aufbau des Gleichgewichtszustandes besitzt eine Halbwertszeit $t_{1 / 2}$, die im allgemeinen ein Vielfaches von der Halbwertszeit $T_{1 / 2}$ für das Abklingen des IS nach Beendigung der Bestrahlung ist (vgl. Tab. 1). Bei Rö.-Strahlen und der Dosisleistung von $1200 \mathrm{r} / \mathrm{min}$ beträgt die Halbwertszeit für den Aufbau des Gleichgewichtes etwa $7 \mathrm{~min} 50 \mathrm{sec}$, bei den $\alpha$-Strahlen der Dosisleistung $700 \mathrm{r} / \mathrm{min} 7 \mathrm{~min} 38 \mathrm{sec}$, während die Halbwertszeit für den Abfall nach Aufhören der Bestrahlung bei den Rö.-Strahlen 6 sec und bei den

len und bei UV viel geringer und beträgt dort nur etwa 10 Prozent. Die Abklingkurve hat, wie eine besondere Untersuchung mit den Röntgenstrahlen ergeben hat, eine von der Dosis praktisch unabhängige Gestalt, und zwar auch nach Anwendung der geringen Dosen des Aufbaugebietes. 


\begin{tabular}{|c|c|c|c|c|c|}
\hline Strahlenart & $\begin{array}{l}\text { Dosisleistung } \\
\text { [rad/min] }\end{array}$ & $\begin{array}{c}\text { Auf baudosis } \\
D_{1} / 2 \\
{[\mathrm{krad}]}\end{array}$ & $\begin{array}{c}\text { Aufbauzeit } \\
t_{1} / 2 \\
\text { [sec] }\end{array}$ & $\begin{array}{c}\text { Grenzwert der } \\
\text { Überlebenden } \\
{[\%]}\end{array}$ & $\begin{array}{c}\text { Abklingzeit* } \\
T_{1} / 2 \\
\text { [sec] }\end{array}$ \\
\hline Röntgen & $\begin{array}{r}12000 \\
1200\end{array}$ & $\begin{array}{r}10,2 \\
9,4\end{array}$ & $\begin{array}{r}50 \\
470\end{array}$ & $\begin{array}{l}52 \\
75\end{array}$ & $\begin{array}{r}12 \\
6\end{array}$ \\
\hline$\alpha$-Strahlen $* *$ & $\begin{array}{r}7000 \\
700\end{array}$ & $\begin{array}{l}8,0 \\
5,5\end{array}$ & $\begin{array}{r}67 \\
458\end{array}$ & $\begin{array}{l}48 \\
73\end{array}$ & $\begin{array}{l}12 \\
6,5\end{array}$ \\
\hline UV & $\begin{array}{r}1800 \mathrm{erg} / \mathrm{cm}^{2} \cdot \min \\
180 \mathrm{erg} / \mathrm{cm}^{2} \cdot \min \end{array}$ & $\begin{array}{l}375 \mathrm{erg} / \mathrm{cm}^{2} \cdot \min \\
270 \mathrm{erg} / \mathrm{cm}^{2} \cdot \min \end{array}$ & $\begin{array}{l}12,5 \\
90\end{array}$ & $\begin{array}{l}43 \\
70\end{array}$ & $\begin{array}{r}11 \\
5\end{array}$ \\
\hline
\end{tabular}

Tab. 1. Zusammenstellung der Versuchsergebnisse. * Für die Differenz Grenzschädigung minus Restschädigung. ** Dosis und Dosisleistung sind an der Agaroberfläche angegeben.

a-Strahlen 6 sec beträgt. Erhöht man die Dosisleistung um den Faktor 10, so ist die Halbwertszeit für den Aufbau bei den Rö.-Strahlen 50 sec, bei den $\alpha$-Strahlen $67 \mathrm{sec}$, während die Halbwertszeit für den Abfall $12 \mathrm{sec}$ ist. Bei UV-Strahlen liegen die Verhältnisse ähnlich, die Aufbauzeit ist gleichfalls länger als die Abklingzeit. Bei der Dosisleistung von $180 \mathrm{erg} / \mathrm{cm}^{2} \mathrm{~min}$ ist die Halbwertszeit für den Aufbau 90 sec und für den Abfall 5 Sekunden. Bei der Dosisleistung von $1800 \mathrm{erg} / \mathrm{cm}^{2} \mathrm{~min}$ ist $t_{1 / 2}$ für den Aufbau etwa zwischen 13 und $11 \mathrm{sec}$ und für den Abfall wiederum 11 Sekunden.

Ebenso müßten sich unter diesen einfachsten Annahmen die Gleichgewichts-Konzentrationen der IS verhalten wie ihre Dosisleistungen, was keineswegs der Fall ist; sie nehmen vielmehr nur sehr langsam mit der Dosisleistung zu.

Es ist daher nur der Schluß möglich, daß die IS nicht nach einer einfachen Eintreffer-Reaktion gebildet werden, sondern durch Mehrtreffer-Reaktionen, d. h. durch wiederholte Reaktionen von langlebigen Radikalen, oder aktiven Molekülen * untereinander. Damit kann dann ohne weiteres erklärt werden, daß der Aufbau der IS viel langsamer vor sich geht als ihr Abklingen nach Aufhören der Bestrahlung. Die Versuchsergebnisse (nur langsamer Anstieg des Gleichgewichtswertes der IS-Konzentration mit steigender Dosisleistung) zeigen weiterhin, daß auch die Vernichtung der IS durch verschiedene Prozesse erfolgt, wobei neben der Vernichtung durch chemisch unveränderten Agar auch Reaktionen der IS mit radiochemisch gebildeten Stoffen von Bedeutung sind. Offensichtlich werden auch verschiedene Arten von IS-Molekülen gebildet, es sind mindestens 2 verschiedene Arten, nämlich mit Halbwertszeiten für das Abklingen $T_{1 / 2}=12 \mathrm{sec}$ und $T_{1 / 2}=6 \mathrm{sec}$ zu unterscheiden **. So kann jedenfalls die Abhängigkeit der Abklingzeiten von der Dosisleistung am einfachsten erklärt werden.

Die Hauptschwierigkeit, welche der soeben skizzierten Auffassung entgegen steht, ist der experimentell bei den kleinen Dosen gefundene praktisch lineare Anstieg der Schädigungsrate mit der Dosis, welcher zunächst nur

* Also von aktiven Molekülen, die durch Zusammenstöße der primär gebildeten Wasserradikale mit den Bestandteilen des Agars entstehen. dadurch erklärbar zu sein scheint, daß die IS-Moleküle nach einer Eintreffer-Reaktion entstehen. Durch eine quantitative Betrachtung kann jedoch leicht gezeigt werden, daß unter bestimmten extremen Bedingungen über die Bildung und Vernichtung auch bei einer Mehrtreffer-Reaktion sich sehr wohl ein praktisch linearer Anstieg der IS-Stoffe mit der Dosis ergeben kann, der dann in einen konstanten Gleichgewichtswert übergeht. Wir gehen dabei von der Voraussetzung aus, daß die IS-Moleküle, nachdem sie sich gebildet haben, die Colizellen (mit einer gewissen Wirkungs-Wahrscheinlichkeit) durch eine einfache Eintreffer-Reaktion abtöten. Streng läßt sich zwar die Berechtigung dieser Annahme nicht beweisen. Sie wird jedoch nahegelegt, da die Schädigungsrate des aufgebrachten $B$. coli nach der Agarbestrahlung grundsätzlich exponentiell mit der Wartezeit abklingt, wie man es nach einer EintrefferReaktion erwartet.

Wir unterscheiden zwischen der Konzentration $N_{1}$ des Vorproduktes der IS-Moleküle und der Konzentration der IS-Moleküle $N_{2}$ selbst. $N_{1}$ wird gebildet durch eine einfache radiochemische Reaktion, d.h. durch Reaktion von Agarbestandteilen mit aktiven Wasserpartikeln. Es ist dann $\mathrm{d} N_{1} / \mathrm{d} r=A$. Die Vernichtung des chemisch aktiven aber ungiftigen Vorproduktes mag in jedem Zeitelement mit einer konstanten Wahrscheinlichkeit erfolgen, z. T. mag sie auch proportional der Dosisleistung vor sich gehen (möglich erscheinen nämlich Reaktionen des Vorproduktes mit unveränderten Agarbestandteilen und mit Produkten radiochemischer Reaktionen, deren Konzentration proportional der Dosisleistung ist). Unter diesen Annahmen ist:

$\frac{\mathrm{d} N_{1}}{\mathrm{~d} r}=A-B N_{1} ; \quad B=B_{1}+\frac{B_{2}}{L} ; \quad N_{1}=\frac{A}{B}\left[1-e^{-B r}\right]$.

Bei der Vernichtungsreaktion des Vorproduktes mag mit einer Wahrscheinlichkeit $\beta$ B ein IS-Molekül entstehen. Die IS verschwindet, wie wir wissen, durch Reaktion mit inaktiven Bestandteilen des Agars. Außerdem wissen wir, $\mathrm{da} ß \mathrm{im}$ Gleichgewichtszustand seine Konzentration $N_{2}$ keineswegs proportional der Dosisleistung

** Da beträchtliche Abweichungen der Abklingkurven von der Exponentialfunktion festgestellt wurden, besteht auch durchaus die Möglichkeit, daß eine ganze Anzahl von IS mit unterschiedlichen Abklingzeiten entstehen. 
ansteigt, sondern noch nicht einmal proportional der Wurzel der Dosisleistung, offenbar können auch die ISMoleküle wiederum durch Zusammenstöße mit radiochemischen Produkten vernichtet werden. Wenn man die Vernichtungs-Wahrscheinlichkeiten pro Zeiteinheit wiederum proportional der Dosisleistung setzt, so ist :

$$
\frac{\mathrm{d} N_{2}}{\mathrm{~d} r}=\beta B N_{1}-N_{2}\left(\frac{C}{L}+D\right) \text {. }
$$

Einsetzen der obigen Gleichung für $N_{1}$ führt zu

$$
\begin{gathered}
\frac{\mathrm{d} N_{2}}{\mathrm{~d} r}=\beta B \frac{A}{B}\left(1-e^{-B r}\right)-C^{x} N_{2}, \\
C^{x}=\frac{C}{L}+D .
\end{gathered}
$$

Die Integration ergibt:

$$
N_{2}=\beta \frac{A}{C^{x}} x\left[1-\frac{e^{-B r} B C^{x}}{B\left(C^{x}-B\right)}-\frac{e^{-C^{x} r} B C^{x}}{C^{x}\left(B-C^{x}\right)}\right]
$$

Man erkennt, daß in den beiden Grenzfällen $C^{x} \gg B$, oder $\mathrm{B} \gg C^{x}$ die IS-Bildung nach der Eintreffergleichung

$$
N_{2}=\beta \frac{A}{C^{x}}\left(1-e^{-B r}\right)
$$

oder

$$
N_{\mathbf{2}}=\beta \frac{A}{C^{x}}\left(1-e^{-C^{\mathbf{x}} r}\right) \quad \text { erfolgt. }
$$

Im ersteren Fall folgt die Konzentration des IS unmittelbar der Konzentration des Vorproduktes (der Größe der Reaktionskonstanten $C^{*}$ wegen). Im 2. Fall stellt sich das Gleichgewicht des Vorproduktes sofort bei Beginn der Bestrahlung ein, und aus diesem Grunde ist die Zeitdauer, die vergeht, bis die GleichgewichtsKonzentration von $N_{2}$ sich ergibt, allein durch den Wert $C^{x}$ gegeben.

Die experimentellen Daten sind nun, wie hier nur kurz angedeutet sei, ausreichend zur Ermittlung der 3 Parameter $B, C, D$, wenn $B$ unabhängig von $L$ angenommen wird. $C$ ist unmittelbar experimentell durch das Abklingen der IS nach Beendigung der Bestrahlung gegeben. $D$ bzw. $C^{x}$ berechnet sich dann aus dem Verhältnis der Gleichgewichts-Konzentration bei den beiden benutzten Dosisleistungen. Durch Vergleich mit den experimentellen Dosiseffektkurven, läßt sich weiter auch $B$ ermitteln. Dabei ergibt sich, daß sowohl bei den energiereichen Strahlen als auch bei UV $C^{x} \gg B$ ist, und zwar zwischen $C^{x}=7,6 B$ und $C^{x}=18,2 B$ liegt. Man erhält mit diesen Zahlenwerten einen zunächst linearen Anstieg für die IS-Konzentration mit der Dosis, wie man es für eine Eintrefferreaktion erwartet. Für den Fall der Anwendung von Röntgenstrahlen ist das Ergebnis der theoretischen Verarbeitung in der Abb. 1 dargestellt.

\section{b) Abhängigkeit der entstehenden IS-Menge vom Peptongehalt des Agars}

Die bisherigen Daten beziehen sich auf Agar mit dem üblichen Peptongehalt von 1 Prozent. Wind Agar mit $10 \%$ Peptongehalt bestrahlt, so findet man eine geringere Schädigungsrate des aufgebrachten B. coli (der
Grenzwert der Schädigungsrate ist etwa auf $1 / 2-1 / 3$ des Grenzwertes bei 1\% Pepton zurückgegangen) und ebenso sind auch die Lebensdauern der IS-Moleküle nach Aufhören der Bestrahlung auf $1 / 2-1 / 3$ der Lebensdauern bei 1\% Pepton reduziert. Durch die Anwesenheit der Peptone (Zusammenstöße der IS-Moleküle mit den Peptonen) wird also die Lebensdauer der IS verkürzt. Die Versuchsdaten schließen jedoch nicht aus, daß die Vorstufe der IS durch Reaktionen des aktiven Wassers mit dem Pepton entsteht.

\section{c) Untersuchungen über die Abhängigkeit} der IS-Bildung vom Sauerstoffgehalt bei den Röntgenund a-Strahlen

Die Agarschicht wurde in ein geschlossenes Gefäß gebracht und einen Tag lang das Gefäß mit Stickstoff oder Sauerstoff durchströmt und dann durch die Gefäßwand hindurch mit den Röntgenstrahlen oder den $\alpha$-Strahlen (im letzteren Fall durch ein $10 \mu$ dickes Hostophanfenster) bestrahlt. Dabei ergab (vgl. Abb. 3) sich, daß die Menge der sich bildenden IS im Falle der Röntgenbestrahlung stark vom $\mathrm{O}_{2}$-Gehalt abhängt, aber bei der Bestrahlung des Agars mit $\alpha$-Strahlen ist das Ergebnis völlig unabhängig vom $\mathrm{O}_{2}$-Gehalt.

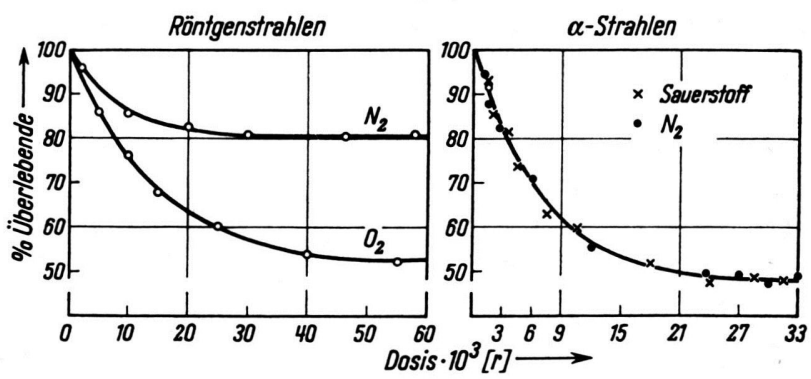

Abb. 3. Bildung der IS im Agar, in Abhängigkeit vom $\mathrm{O}_{2}$-Gehalt.

Qualitativ verhält sich also bezüglich der Abhängigkeit vom $\mathrm{O}_{2}$-Gehalt die Erzeugung der IS genauso wie die Erzeugung des $\mathrm{H}_{2} \mathrm{O}_{2}$ in reinem Wasser, auch dort ist die $\mathrm{H}_{2} \mathrm{O}_{2}$-Bildung bei Anwendung von Röntgenstrahlen in stärkstem $\mathrm{Maße}$ abhängig vom $\mathrm{O}_{2}$-Gehalt, während bei Anwendung von $a$-Strahlen die $\mathrm{H}_{2} \mathrm{O}_{2}$-Bildung in Abhängigkeit vom $\mathrm{O}_{2}$-Gehalt geringfügig ist. Ein einfacher Versuch zeigt jedoch, daß bei der Agarbestrahlung es sich sicher nicht um eine primäre Bildung von $\mathrm{H}_{2} \mathrm{O}_{2}$ handelt ${ }^{*}$ bzw. das IS nicht erst sekundär durch Reaktionen des $\mathrm{H}_{2} \mathrm{O}_{2}$ mit dem Agar gebildet wird. Bei den angewandten Dosen bis zu etwa $5000 \mathrm{rad}$ wird in $\mathrm{O}_{2}$-haltigem Wasser $5,7 \cdot 10^{-8} \mathrm{~g} / \mathrm{cm}^{3} \quad \mathrm{H}_{2} \mathrm{O}_{2}$ gebildet. Auch noch viel höhere Konzentrationen von $\mathrm{H}_{2} \mathrm{O}_{2}$ erzeugen keinerlei IS in dem Agar. Selbst wenn man den Agar mit einer $\mathrm{H}_{2} \mathrm{O}_{2}$-Lösung von 600-mal größerer Konzentration als die angegebene durchfeuchtet und

\footnotetext{
* Ebenso wie bei der unmittelbaren Bestrahlung von B. coli mit energiereichen Strahlen, nach den in der Literatur angegebenen analogen Versuchen.
} 
anschließend B. coli aufbringt, stellt man keinerlei Anzeichen einer Inaktivierung fest.

Es hat sich also in der Tat ergeben, daß ebenso wie bei der direkten Bestrahlung von $B$. coli mit $\alpha$-Strahlen die Erzeugung der diffusiblen IS unabhängig von der $\mathrm{O}_{2}$ Konzentration ist. Es bleibt nur noch übrig, den Nachweis zu führen, daß die Ionenausbeute für die IS-Bildung bei Anwendung von $\alpha$-Strahlen größer ist als bei der Anwendung von Röntgenstrahlen, und zwar auch bei Anwesenheit von Sauerstoff. Unter Ionenausbeute der IS-Bildung verstehen wir dabei die pro $100 \mathrm{eV} / \mathrm{cm}^{3}$ im Agar bei kleinen Dosen (Gebiet des linearen Anstieges mit der Dosis) gebildeten IS-Moleküle.

\section{d) Die Diffusionslänge des IS im Agar und die relativen Ionenausbeuten bei seiner Erzeugung durch energiereiche Strahlen}

Die Bestimmung der Ionenausbeute bzw. ihrer relativen Werte ist nur möglich, wenn die Diffusionslänge der IS in Agar bekannt ist, und damit auch die Agartiefe, aus der heraus das IS noch zu den Colibakterien gelangen kann.

$\mathrm{Zu}$ diesem $\mathrm{Zwecke}$ wurden durch ein geeignetes Schnittverfahren (unterkühlter Agar wurde mit einem Mikrotom geschnitten) Agarschichten verschiedener Dicke, nämlich zwischen 20 und $440 \mu$ Dicke hergestellt und diese Schichten auf einer Al-Unterlage mit den Rö.-Strahlen bestrahlt. Es wurde dann in der üblichen Weise die Schädigung der unmittelbar nach der Bestrahlung aufgebrachten Colizellen bestimmt. Dabei ergab sich (vgl. Abb. 4) eine zunächst lineare Zunahme der Schädigung mit steigender Schichtdicke des Agars bis zu dem Grenzwert der Schädigung von etwa $50 \%$ des aufgebrachten B. coli, und zwar ist die Halbwertsschicht, bei der man also 25\% Schädigung erreicht, etwa $78 \mu$ dick. Dadurch ist also die Agartiefe gegeben, aus der heraus die IS zu den Colizellen an der Oberfläche zu diffundieren vermögen. Im Gegensatz hierzu können bei Anwendung von $\alpha$-Strahlen sämtliche innerhalb der a-Reichweite in Agar gebildeten IS-Molekülen zu den Colizellen diffundieren *. Die weitere Auswertung des Versuches geht in folgender Weise vor sich:

Wir betrachten eine Colizelle an der Agaroberfläche mit dem Radius $\varrho$. Ein im Abstand $R$ im Agar gebildetes IS-Molekül gelangt mit der Wahrscheinlichkeit $W=(\varrho / R) e^{-(R-\varrho) / l}$, wobei $l$ als Diffusionslänge bezeichnet wird zur Colizelle ${ }^{* *}$. Wenn $A$ IS-Moleküle pro $\mathrm{cm}^{3}$ gebildet werden, so gelangen aus einer Schicht in der Tiefe $d$ und der Dicke $\Delta \quad W=A 2 \pi \varrho l e^{-(d-\varrho) / l} d \Delta$

* Das heißt, die Schädigungswahrscheinlichkeit der Colizellen durch ein IS-Molekül ist bei den $a$-Strahlen nur von der Integraldosis abhängig und unabhängig davon, wie weit das $a$-Teilchen in den Agar eindringt. Dies haben besondere Versuche gezeigt.

${ }^{* *}$ Literatur zu dieser Formel vgl. l. c. ${ }^{1}$ oder l. c. ${ }^{8}$. Die folgenden Formeln ergeben sich ohne weiteres durch elementare räumliche Integration über die Grundformel, wobei immer $\varrho \ll l$ angenommen ist.

8 E. Six, Z. Naturforschg. 9 b, 272 [1954].

*** Bei gleichmäßiger Verteilung von Aktivität in einem beliebigen Körper ist die Dosisleistung nach einer bekann-

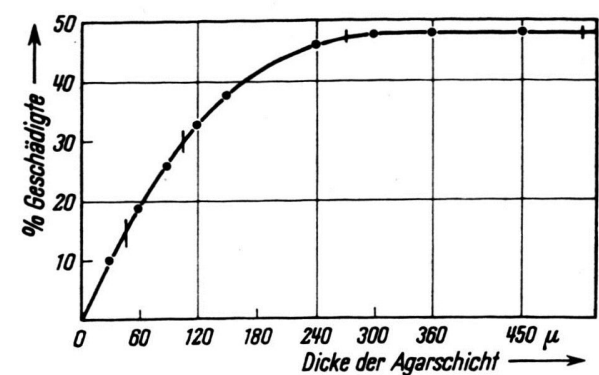

Abb. 4. Geschädigte Coli-Bakterien als Funktion der Agarschichtdicke bei Röntgen-Bestrahlung.

IS-Moleküle zur betrachteten Colizelle. Die Zahl der insgesamt aus der Oberflächenschicht von der Dicke $d$ zur Zelle gelangenden Teilchen ist:

$$
W=A 2 \pi \varrho l^{2}\left(1-e^{-d / l}\right) \text {. }
$$

Die Zahl der insgesamt aus einer Schicht der Tiefe $d \gg l$ zu einer bestimmten Colizelle diffundierenden IS-Moleküle ist hiernach bei Rö.-Strahlen $W=A 2 \pi \varrho l^{2}$.

Die Zahl der aus einer Schicht $d \ll l$ zur Zelle diffundierenden IS-Moleküle ist

$$
W=A d 2 \pi \varrho l,
$$

im Falle der $a$-Strahlen (Plattenpräparat) ist an Stelle von $A d$ die insgesamt im Agar pro $\mathrm{cm}^{2}$ Agaroberfläche erzeugte IS-Menge zu setzen.

Der Vergleich wird durchgeführt für Dosisleistungen, welche im Agar zu einer Grenzschädigung von 50\% führen. Bei den $\alpha$-Strahlen ist dies der Fall bei einer Dosisleistung an der Agaroberfläche von etwa $9000 \mathrm{rad} / \mathrm{min}$ und die Integraldosis $I_{1 / 2}$ pro $\mathrm{cm}^{2}$ Oberfläche, die zur Hälfte der Grenzschädigung führt, ist *** $0,6 \cdot \frac{0,1}{2} \cdot 5 \cdot 40=6$ gr rad. Bei den Röntgenstrahlen beträgt die entsprechende Halbwertsdosis für den Aufbau der IS etwa $10000 \mathrm{rad}$, also der Wert von $D_{1 / 2} \cdot l$ ist $10^{4} \cdot 78 \cdot 10^{-4}=78 \mathrm{gr} \mathrm{rad}$. Mithin ist das Verhältnis der Ionenausbeute $a_{\mathrm{R}}$ bei den Röntgenstrahlen zu $a_{a}$ bei den $\alpha$-Strahlen.

$$
\frac{a_{\mathrm{R}}}{a_{a}}=\frac{I_{1 / 2} 2 \pi \varrho l}{D_{1 / 2} 2 \pi \varrho l^{2}}=\frac{I_{1 / 2}}{D_{1 / 2} l}=\frac{6}{78} \approx \frac{1}{10} .
$$

Wir gelangen also zu dem Ergebnis, daß die Ionenausbeute ${ }^{* * * *}$ bei Anwendung der $\alpha$-Strahlen etwa 10-mal so groß wie bei Anwendung der Rö.-Strahlen ist. Es hat sich mithin eine weitgehende Übereinstimmung zwischen

ten Formel $0,6 \cdot E \cdot \frac{\mathrm{mC}}{\mathrm{gr}} \frac{\mathrm{rad}}{\mathrm{sec}}(E$ in $\mathrm{MeV})$; es ist weiter zu berücksichtigen, daß nur die Hälfte der $a$-Strahlen in den Agar eindringt. Experimentell ergaben sich bei einer Präparatstärke von $0,1 \mathrm{mC} / \mathrm{cm}^{2}$ (dies liefert and-Agaroberfläche $9000 \mathrm{rad} / \mathrm{min}$ ) $t_{1 / 2}=40$ Sekunden. $E$ ist bei Polonium $=5 \mathrm{MeV}$.

*** Unter Ionenausbeute der IS-Bildung im Agar verstehen wir also die Größe, die in der obigen kinetischen Ableitung durch $\beta \cdot \frac{A B}{C^{*}}$ bezeichnet ist, bzw. ihr proportional ist. 
der Bildung der IS im Agar und der von Dobson angenommenen Bildung von diffusiblen IS in der Colizelle bei direkter Bestrahlung ergeben. Es ist nämlich in beiden Fällen die IS-Entstehung bei Anwendung der

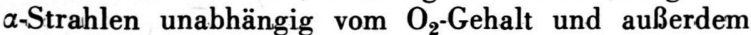
ist bei den $a$-Strahlen die Ionenausbeute für die ISEntstehung größer als bei den Rö.-Strahlen.

Wir verfolgen die Analogie mit den Ergebnissen von Dosson weiterhin, indem wir die Absolutzahl der aus dem Agar zu den Colizellen diffundierenden IS-Moleküle betrachten.

Die Zahl der primären Ionisationen pro $\mu^{3}$ bei der Dosis $1 \mathrm{rad}$ ist etwa von der Größe 1 , also gelangen bei einer Ionenausbeute $a_{\mathrm{R}}$ (bezogen auf die Zahl der primären Ionisationen *) und bei der Röntgenstrahlendosis von $10000 \mathrm{rad}$ zu einer bstimmten Colizelle, wenn wir diese wiederum kugelförmig mit dem Radium $\varrho=1 \mu$ annehmen, $a_{\mathrm{R}} \cdot 10^{4} \cdot 2 \pi \cdot 1 \cdot 78^{2}, \mathrm{~d}$, h. ungefähr $a_{\mathrm{R}} \cdot 4 \cdot 10^{8}$ IS-Moleküle. Die Vorbestrahlung des Agars mit $10000 \mathrm{rad}$ führt jedoch nur zu einer Schädigungsrate von 25 Prozent. Die Inaktivierung der Zelle erfordert also die Diffusion von ungefähr $a_{\mathrm{R}} \cdot 10^{9}$ IS-Molekülen zur Zelle. $a_{\mathrm{R}}$ ist dabei gegeben durch $\beta A B / C^{*}$ unserer obigen kinetischen Herleitung. Da das Vorprodukt mit einer Ionenausbeute von höchstens etwa 1 gebildet werden dürfte aber $B / C^{*}$ etwa $1 / 10$ ist, ist $a_{a}$ für $\alpha$-Strahlen $<1 / 10$ und $a_{\mathrm{R}}$ für Röntgenstrahlen $<1 / 100$ und die Wirkungs-Wahrscheinlichkeit $b$ eines IS-Moleküls, welches zur Colizelle gelangt $b=\frac{1}{a_{\mathrm{R}} \cdot 10^{9}}>10^{-7}$.

Zur Zeit kann leider nicht mit Sicherheit ausgeschlossen werden, daß bei den von Dosson angewandten direkten Bestrahlungen neben den direkten Korpuskeldurchgängen durch die Colizelle auch im Agar erzeugte IS an der Schädigung beteiligt waren. $\alpha$-Strahlenversuche von Dosson, bei denen durch Vorschalten von Filtern die Reichweite der $\alpha$-Strahlen und damit ihre Eindringtiefe im Agar variiert wurde, liefern keinen unmittelbaren Hinweis auf eine ins Gewicht fallende Mitwirkung von im Agar entstandenen IS. Machen wir entsprechend die Annahme, daß praktisch nur die Ionisationen in der Colizelle bei den direkten Bestrahlungen wirksam werden, so ergibt sich bei den Deuteronenversuchen von Dosson für die Zahl der in der Colizelle bei der Abtötung entstehenden IS-Moleküle $a \cdot 10^{3}$ ( $a$ Ionenausbeute, rund jeder 50 . Deuteronendurchgang inaktiviert und erzeugt dort rund 200 primäre Ionisationen). Diese Zahl ist also sechs Zehnerpotenzen kleiner als die entsprechende für die Inaktivierung durch IS nach Vorbestrahlung des Agars. Daraus würde also folgen, daß die Dobsonschen IS mit einer größeren Ionenausbeute gebildet werden und eine weit größere Inaktivierungs-Wahrscheinlichkeit besitzen als die im Agar

\footnotetext{
* Praktisch identisch mit $100 \mathrm{eV}$.

9 A. Eucken, Chemische Physik 1944 B II, 2, S. 1067 (Tabelle).

** Es ist schwer, eine Fehlergrenze für das gefundene Mol.Gew. anzugeben, sie mag $\pm 20 \%$ betragen, so daß $M=200 \pm 40$ ist. Der von uns gefundene Wert für den Diffusionskoeffizienten stimmt praktisch mit seinem Wert
}

entstehenden IS, also Teilchen mit einer weit größeren Reaktionsfähigkeit und viel geringeren Lebensdauer bzw. Diffusionslänge darstellen. Die Aufteilung der Primäreffekte bei der direkten Bestrahlung von B. coli auf Agar, in solche, die von Korpuskeldurchgängen durch die Colizelle herrühren und in solche, die durch in Agar entstandene IS bedingt sind, erfordert jedoch noch ausgedehnte Spezialuntersuchungen. (Diese sind bereits im Gang.)

Eine Berechnung des mittleren Mol.-Gew., welches die im Agar erzeugte IS besitzen muß, ergibt sich folgendermaßen: für die Diffusionslänge $l$ besteht die Beziehung $l=\sqrt{D_{\mathrm{i}} T_{1 / e}}$ wobei $D_{\mathrm{i}}$ der Diffusionskoeffizient ist und $T_{1 / e}$ die Zeit darstellt, in der bei den Bedingungen, unter denen $l$ gemessen ist, die Konzentration des IS auf $1 / e$ abfällt. Man kann also $D_{\mathrm{i}}$ berechnen und hieraus auf die Masse des IS-Moleküls schließen.

Es ist $D_{\mathrm{i}}=l^{2} / T_{1 / e} ; l$ ist bestimmt (vgl. Abb. 4) zu $78 / 0,69=115 \mu$ und $T_{1 / e}$ ist unter gleichen Bedingungen 22 Sekunden. Für $D_{\mathrm{i}}$ erhält man also den Wert $D_{\mathrm{i}}=115^{2} \cdot 10^{-8} / 22=0,6 \cdot 10^{-5} \mathrm{~cm}^{2} / \mathrm{sec}$. Den Diffusionskoeffizient von $1,2 \cdot 10^{-5} \mathrm{~cm}^{2} \mathrm{sec}$ besitzt in Wasser das Harnstoffmolekül mit dem Mol.-Gew. 60 (vgl. l. c. ${ }^{9}$ ). Da $D_{\text {i }}$ etwa mit $\sqrt{1 / M}$ variiert, erhält man für das Mol.Gew. des IS die Größenordnung 240. Es ist bemerkenswert, daß die von uns untersuchte IS ein Mol.-Gew. von der gleichen Größe wie das Peroxyd des Disuccinoyls $(M=240)$ besitzt, welches nach Latarjet (vgl. l. c. ${ }^{5}$ Latarjet 1959) unter den bisher untersuchten Peroxyden das biologisch wirksamste ist **.

Es ist daher lehrreich, die Wirkungs-Wahrscheinlichkeiten (bzw. die Diffusionszahlen) die wir für die strahleninduzierte IS gefunden haben, mit der WirkungsWahrscheinlichkeit von Disuccinoylperoxyd zu vergleichen.

Nach einer Angabe von Latarjet überleben $30 \%$ des B. coli (vom Stamm B), wenn es in einer Suspension 30 min lang einer Succinoylperoxyd-Konzentration von $10^{-5}-m$. ausgesetzt wird. Die Zahl der in dieser Zeit zu den Colizellen gelangenden Peroxyd-Moleküle berechnet sich nach der oben bereits angewandten Formel zu $4 \pi N \varrho D_{\mathrm{i}} T=4 \pi 6 \cdot 10^{15} \cdot 10^{-4} \cdot 10^{-5} \cdot 1800 \approx 10^{11}$.

Mithin wären also die Wirkungs-Wahrscheinlichkeiten der im Agar durch die Strahlung erzeugten IS um 4 Größenordnungen höher als die Wirkungs-Wahrscheinlichkeit des Peroxyds. Bei der Beurteilung dieses Ergebnisses ist allerdings noch zu bedenken, daß die Angabe von Latarjet sich auf eine Colisuspension im Ruhezustand bezieht, welche gegen organische Peroxyde viel unempfindlicher ist als eine Suspension im Wachstum, doch dürfte sich hierdurch die Wirkungs-Wahrscheinlichkeit höchstens auf $1 / 10$ vermindern.

für Mannit $(M=182)$ überein. Das Disuccinylperoxyd zerfällt in Wasser noch in zwei annähernd gleich große Bruchstücke. Ein schwer bestimmbarer Fehler ergibt sich daraus, daß entgegen den Annahmen unserer Rechnungen $l$ durch Versuche mit in der Tiefe begrenzten Agarschichten gewonnen wurde. 
3. Versuche überdie Wirkung von vorbestrahltem Agar auf Hefezellen

Nach unseren einleitenden Ausführungen ist anzunehmen, daß die Wirkungs-Wahrscheinlichkeiten der ISMoleküle bei der Inaktivierung von Colizellen sehr viel größer sind als bei der Inaktivierung von Hefezellen. In der Tat erzielten wir nach Vorbestrahlung des Agars mit $\alpha$ - und Röntgenstrahlen in keinem Fall nachweisbare Inaktivierung der Hefezellen. Da bei der Bestrahlung des Agars mit steigenden Dosen sich ein Gleichgewichtswert der IS-Konzentration ausbildet, der nur 30-50\% der Colizellen zur Abtötung bringt, anderenseits aber die $D_{1 / 2}$ der Hefezellen um etwa $50 \%$ höher liegt als für die Colizellen, war dieser Befund auch für den Fall zu erwarten, daß die Wirkungs-Wahrscheinlichkeiten der IS-Moleküle bei der Hefe nur unwesentlich geringer sind als bei Colibakterien. Wie aus der Abb. 1 hervorgeht, hat jedoch ein relativ geringer Prozentsatz der ISMoleküle viel längere Lebensdauern als die von uns bisher untersuchten IS-Moleküle und nach den Angaben

* Es ist uns mit den uns zur Verfügung stehenden Mitteln nicht geglückt, durch Bestrahlung des Agars mit Röntgenstrahlen eine höhere Schädigungsrate des B. coli als 50\% zu erzielen, selbst bei einer Dosisleistung von $200000 \mathrm{rad}$ pro Minute.

Bei der Bewertung der Unempfindlichkeit der Hefezellen gegen die im Agar entstandenen IS muß noch in der Literatur (insbesondere STEIN und HARM $^{6}$ ) war zu erwarten, daß durch Anwendung von sehr viel höheren Dosen sich diese IS-Moleküle mit weit höheren Lebensdauern in viel höheren Konzentrationen herstellen lassen, als die kurzlebigen IS. In der Tat konnten wir bei der Anwendung von UV durch Bestrahlung des Agars mit 500-mal höheren Dosen (100-mal längere Bestrahlungszeiten) erreichen, daß 95\% des nach der Bestrahlung aufgebrachten $B$. coli geschädigt wird. Bringt man jedoch nach der gleichen Vorbestrahlung des Agars Hefezellen auf, so liegt die Erhöhung der Schädigungsrate sicher innerhalb einer Nachweisgrenze etwa von 5 und 10 Prozent. Aus dem Versuch geht hervor, daß die Wirkungs-Wahrscheinlichkeit der im Agar induzierten IS-Moleküle bei den Colizellen mindestens 500-mal größer ist als bei den Hefezellen *. (Dabei ist für die Hefezelle ein 5-mal größerer Durchmesser als für die Colizelle in Rechnung gesetzt.)

Wir danken der Deutschen Forschungs g e m e i n s c h a f $\mathrm{t}$ für die Unterstützung der Arbeit.

daran gedacht werden, daß vielleicht in der pflanzlichen Zellmembran der Hefezellen eine Entaktivierung der IS mit viel größerer Wahrscheinlichkeit erfolgen kann als in der Membran der Colizelle. Das Ergebnis des Versuches allein besitzt daher keine Beweiskraft bezüglich der Unwirksamkeit der diffusiblen IS bei den Hefechromosomen.

\title{
Interaction and mixed aggregation of proteins from Tobacco mosaic virus strains
}

\author{
By Satyabrata Sarkar \\ Aus dem Max-Planck-Institut für Biologie, Abt. Melchers, Tübingen, Corrensstraße 41 \\ (Z. Naturforschg. 15 b, 778-786 [1960]; eingegangen am 10. Oktober 1960)
}

\begin{abstract}
Proteins from four strains of TMV, namely vulgare, flavum, dahlemense and Holmes' rib grass, were electrophoretically examined singly and in pairs at different hydrogen ion concentrations.

In weakly alkaline media an average of six polypeptide chains of TMV-protein remain associated together, while constant dissociation and reassociation takes place. This dynamic state of equilibrium is responsible for transient reciprocal associations of proteins or polypeptide chains from the first three TMV-strains. On lowering the $p_{\mathrm{H}}$ value, these interacting proteins form mixed aggregates with intermediate mobilities. Protein from the fourth strain, Holmes' rib grass, when tested against the strain, vulgare, neither showed any interaction in alkaline media nor formed mixed aggregates.

Factors determining the formation of mixed aggregates and the possible relationship among the
\end{abstract} four strains have been discussed.

The protein-part of a single Tobacco mosaic virus particle consists of $2130 \pm 40$ polypeptide chains which are very probably identical with one another ${ }^{1}$ (Franklin, Caspar and Klug, 1959). A polypeptide chain of the common TMV strain, vulgare, has a molecular weight of about 17500 and consists of

1 R. E. Franklin, D. L. D. Caspar and A. Klug, in: Plant Pathology, Problems and Progress 1908-1958, Univ. of Wisconsin, Pp. 447-461 [1959].
$157^{*}$ amino acids (Wittmann and Braunitzer, 1959) ${ }^{2}$ whose sequence has been recently worked out ${ }^{3}$ (ANDERER et al., 1960).

* According to a recent letter from Professor W. M. Stanley addressed to Professor G. Melchers a polypeptide chain of TMV protein contains 158 amino acids and not 157 as found here earlier. This result could now be confirmed also by Dr. H. G. Wittmann.

2 H. G. Wittmann and G. Braunitzer, Virology 9, 726 [1959].

3 A. Anderer, H. Uhlig, E. Weber and G. Schramm, Nature [London] 186, 922 [1960]. 